\title{
Modeling abundances in galaxies
}

\author{
Chiaki Kobayashi ${ }^{1}$ and Philip Taylor ${ }^{2}$ \\ ${ }^{1}$ Centre for Astrophysics Research, Science and Technology Research Institute, University of \\ Hertfordshire, Hertfordshire, AL10 9AB, UK; email: c.kobayashi@herts .ac.uk \\ ${ }^{2}$ Research School of Astronomy \& Astrophysics, Australian National University, Mt. Stromlo \\ Observatory, Cotter Rd., Weston, ACT 2611, Australia
}

Abstract. We reviewed chemical evolution of galaxies using our cosmological simulations.

Keywords. galaxies: abundances, galaxies: evolution, supernovae: general

\section{Introduction}

Heavy elements are produced from various types of supernovae (and asymptotic giant branch stars). Nucleosynthesis yields have been relatively well understood (Nomoto et al. 2013 for a review), and therefore we can use elemental abundances and isotopic ratios to constrain the formation and evolutionary histories of galaxies. This approach is called galactic archaeology.

Despite more than ten years of surveys, no stars that have an elemental abundance pattern consistent with pair-instability supernovae (PISNe) have been found. Instead, the elemental abundance patterns of extremely metal-poor (EMP) stars and metal-poor damped Lyman $\alpha$ systems, in particular the large carbon enhancement, are well reproduced by faint supernovae (Nomoto et al. 2013). Some EMP stars show low $[\alpha / \mathrm{Fe}]$ ratios, which can be explained with low-mass $\left(\sim 13-15 M_{\odot}\right)$ supernovae (Kobayashi et al. 2014). The low $[\alpha / \mathrm{Fe}]$ ratios and their trends in dwarf spheroidal galaxies can be explained by subclasses of Type Ia supernovae (SNe Ia) such as SN 2002cx-like objects and sub-Chandrasekhar mass explosions (Kobayashi et al. 2015). Although these variation in supernovae is important in the early Universe or metal-poor systems such as dwarf spheroidal galaxies (and is interesting in the point of view of stellar astrophysics), the chemical enrichment history of galaxies is mainly determined from "major" chemical enrichment sources, i.e., core-collapse supernovae/hypernovae and SNe Ia.

Chemical enrichment of interstellar medium depends on the local conditions (e.g., gas density), and therefore the global timescale depends on the environment or galaxy mass. Although chemical evolution of galaxies has been modelled with one-zone or semianalytic approach, chemo-dynamical simulations have an advantage in calculating such inhomogeneous chemical enrichment. In Milky Way-type simulations, the bulge stars have formed during the assembly of gas-rich sub-galaxies at $z \gtrsim 3$, and have older age and higher $[\alpha / \mathrm{Fe}]$ than in the disk (Kobayashi \& Nakasato 2011). Massive galaxies have quenched star formation by feedback from active galactic nuclei (AGN), and tend to have high metallicities and high $[\alpha / \mathrm{Fe}]$ (Taylor \& Kobayashi 2015a). These simulations are also the best to predict supernova/hypernova/gamma-ray burst rates and the properties of their host galaxies.

\section{Abundances in galaxies}

We show chemical evolution of galaxies in our cosmological, chemodynamical simulations (Taylor \& Kobayashi 2015a) that include relevant physical processes such as star 

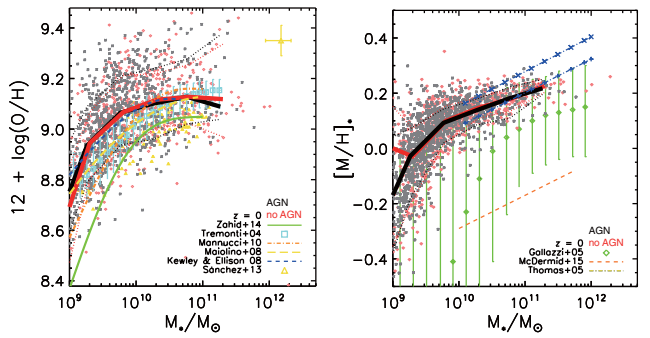

Figure 1. Present mass-metallicity relations for gas (left panel, SFR weighted) and stars (right panel, V-band luminosity weighted). Simulated data are shown by the black stars (with AGN) and red diamonds (without AGN), with their median (solid lines) and $1 \sigma$ scatter (dotted lines) overlaid. Also shown are the observational data (see Taylor \& Kobayashi 2015a for the references).
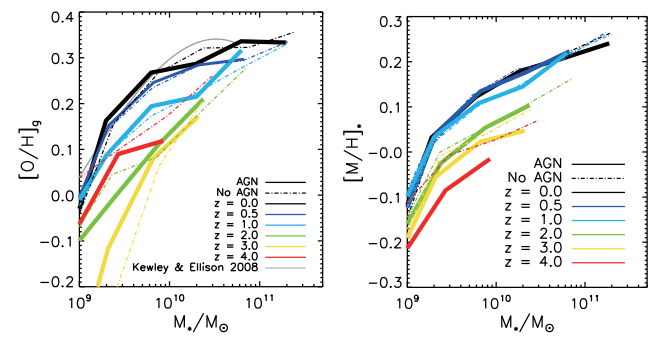

Figure 2. Time evolution of mass-metallicity relations for gas (left panel, SFR weighted) and stars (right panel, V-band luminosity weighted) from $z=0$ to 5 . Solid (dot-dashed) lines correspond to the simulation with (without) AGN feedback.

formation, chemical enrichment, and feedback from supernovae (Kobayashi et al. 2007) and from AGN (Taylor \& Kobayashi 2014). Different from previous works, our black holes are not the products of galaxy mergers but originate from the first stars, motivated by the lack of the signature of PISNe. Our AGN feedback causes metal-enhanced outflows, which transport metals into the circumgalactic medium and the intergalactic medium (Taylor \& Kobayashi 2015b).

Nonetheless, the metallicity changes of galaxies are negligible, and the mass-metallicity relations (MZRs), which are mainly generated by supernova feedback at the first star burst (Kobayashi et al. 2007), are preserved. Figure 1 shows the present MZRs of simulated galaxies, which are in good agreement with various observations, both for gas-phase abundances and stellar populations. We also show the time evolution of these MZRs in Figure 2. The stellar MZR does not change its shape, but the metallicity significantly increases from $z \sim 2$ to $\sim 1$, while the gas-phase MZR does change shape, having a steeper slope at higher redshifts $(z \lesssim 3)$. Within galaxies, metallicity radial gradients are produced; the metallicities of central parts of galaxies are always higher than in the outskirts. We find a weak correlation between the gradients and galaxy mass, which is consistent with available observations such as ATLAS ${ }^{3 \mathrm{D}}$. Stellar metallicity gradients are found to be made shallower by galaxy mergers, and are generally shallower in denser environments, regardless of galaxy mass. On the other hand, gas-phase metallicity gradients are affected more strongly by AGN feedback in massive galaxies, and stellar feedback in low-mass galaxies. In principle, it is possible to constrain physical processes during galaxy evolution from these two gradients.

\section{References}

Kobayashi, C., Ishigaki, M. N., Tominaga, N., \& Nomoto, K. 2014, ApJ, 5, L5

Kobayashi, C. \& Nakasato, N. 2011, ApJ, 729, 16

Kobayashi, C., Nomoto, K., \& Hachisu, I. 2015, ApJ, 804, L24

Kobayashi, C., Springel, V., \& White, S. D. M. 2007, MNRAS, 376, 1465

Nomoto, K., Kobayashi, C., \& Tominaga, N. 2013, ARA\&A, 51, 457

Taylor, P. \& Kobayashi, C. 2014, MNRAS, 442, 2751

Taylor, P. \& Kobayashi, C. 2015a, MNRAS, 448, 1835

Taylor, P. \& Kobayashi, C. 2015b, MNRAS, 452, L59 